\title{
A!
}

This is an electronic reprint of the original article.

This reprint may differ from the original in pagination and typographic detail.

Setälä, Tero; Shevchenko, Andriy; Kaivola, Matti; Friberg, Ari

\section{Polarization time and length for random optical beams}

Published in:

Physical Review A

DOI:

10.1103/PhysRevA.78.033817

Published: 11/09/2008

Document Version

Publisher's PDF, also known as Version of record

Please cite the original version:

Setälä, T., Shevchenko, A., Kaivola, M., \& Friberg, A. (2008). Polarization time and length for random optical beams. Physical Review A, 78(3), 1-6. [033817]. https://doi.org/10.1103/PhysRevA.78.033817

This material is protected by copyright and other intellectual property rights, and duplication or sale of all or part of any of the repository collections is not permitted, except that material may be duplicated by you for your research use or educational purposes in electronic or print form. You must obtain permission for any other use. Electronic or print copies may not be offered, whether for sale or otherwise to anyone who is not an authorised user. 


\title{
Polarization time and length for random optical beams
}

\author{
Tero Setälä, ${ }^{1, *}$ Andriy Shevchenko, ${ }^{1}$ Matti Kaivola, ${ }^{1}$ and Ari T. Friberg ${ }^{1,2, \dagger}$ \\ ${ }^{1}$ Department of Engineering Physics, Helsinki University of Technology (TKK), P.O. Box 3500, FI-02015 TKK, Finland \\ ${ }^{2}$ Department of Physics and Mathematics, University of Joensuu, P.O. Box 111, FI-80101 Joensuu, Finland
}

(Received 5 June 2008; published 11 September 2008)

\begin{abstract}
We investigate the dynamics of the instantaneous polarization state of stationary, partially polarized random electromagnetic beamlike fields. An intensity-normalized correlation function of the instantaneous Poincaré vector is introduced for the characterization of the time evolution of the polarization state. This polarization correlation function enables us to define a polarization time and a polarization length over which the polarization state remains substantially unchanged. In the case of Gaussian statistics, the polarization correlation function is shown to assume a simple form in terms of the parameters employed to characterize partial coherence and partial polarization of electromagnetic fields. The formalism is demonstrated for a partially polarized, temporally Gaussian-correlated beam, and black-body radiation. The results are expected to find a range of applications in investigations of phenomena where polarization fluctuations of light play an important role.

DOI: 10.1103/PhysRevA.78.033817

PACS number(s): 42.25.Ja, 42.25.Kb
\end{abstract}

\section{INTRODUCTION}

The central parameters of optical coherence theory that quantify the spatial and temporal coherence properties and partial polarization of fluctuating optical fields are customarily formulated either for scalar waves or for electromagnetic beamlike (two-dimensional) fields [1-3]. The traditional degree of coherence, and the related coherence time and length, describe the capability of a beam to interfere, but are strictly valid only for scalar waves. The concept of the degree of polarization characterizes the partial polarization of an electromagnetic beam, and the Stokes parameters and the Poincaré sphere provide an elegant geometrical depiction of the state of polarization. Recently, considerable attention has been paid to the coherence properties of electromagnetic fields and the characterization of partial polarization of general, three-dimensional optical fields. In particular, the concept of the degree of coherence has been extended to both paraxial and nonparaxial electromagnetic fields $[4,5]$ (see also Ref. [6]), and the degree of polarization has been generalized to nonparaxial electromagnetic fields [7-9]. Despite the recent developments in electromagnetic coherence theory, the time evolution of the instantaneous polarization state of an optical field, described by the instantaneous Poincaré vector, has not been much considered in the past. The fluctuation-induced changes in the polarization state, however, are an inherent property of partially polarized fields, and their investigation would provide additional information on the properties of the source that generates the field and the medium in which the field propagates.

In this work we investigate the dynamics of the polarization state of statistically stationary, partially polarized beamlike electromagnetic fields. The aim of the work is to intro-

\footnotetext{
*Tero.Setala@tkk.fi

${ }^{\dagger}$ Also at the Department of Microelectronics and Applied Physics, Royal Institute of Technology (KTH), Electrum 229, SE-164 40 Kista, Sweden.
}

duce a quantity that describes the similarity of polarization states at two instants of time. From this quantity one can deduce a characteristic time over which the instantaneous polarization state remains essentially unchanged. In analogy with the traditional coherence time and length, one can then determine a polarization length as the distance traveled by light within the polarization time. The formulation is general, but here we apply it to fields obeying Gaussian statistics. For such fields, the polarization correlation function takes on a simple and compact form in terms of the degree of polarization and two other quantities that measure the electromagnetic coherence. We apply the theory to a partially polarized, temporally Gaussian-correlated beam and black-body radiation.

In Sec. II we recall the measures that describe the coherence and polarization properties of random electromagnetic beams. Next, in Sec. III, the polarization correlation function is introduced and written for the case of Gaussian statistics. Furthermore, the behavior and properties of the polarization correlation function are presented, and the polarization time and length are defined. Then in Sec. IV, the formalism is demonstrated with specific examples. Finally, Sec. V summarizes the work.

\section{CHARACTERIZATION OF ELECTROMAGNETIC COHERENCE}

Consider a fluctuating, stationary electromagnetic beam propagating in the $z$ direction in free space. At a space-time point $(\mathbf{r}, t)$ the electric vector of such a field is represented by a zero-mean complex analytic signal $\mathbf{E}(\mathbf{r}, t)$ $=\left(E_{x}(\mathbf{r}, t), E_{y}(\mathbf{r}, t)\right)$, a vector transverse to the propagation direction. At a pair of points $\mathbf{r}_{1}$ and $\mathbf{r}_{2}$, and at time difference $\tau$, the second-order coherence properties of the field are described by the (electric) $2 \times 2$ mutual coherence matrix [1]

$$
\mathcal{E}\left(\mathbf{r}_{1}, \mathbf{r}_{2}, \tau\right)=\left\langle\mathbf{E}^{*}\left(\mathbf{r}_{1}, t\right) \mathbf{E}\left(\mathbf{r}_{2}, t+\tau\right)\right\rangle .
$$

In this equation, the angle brackets and the asterisk denote averaging and complex conjugation, respectively. We assume 
that the field is ergodic and, therefore, the averaging can be taken either as an ensemble average or a time average over a single realization of the field. From Eq. (1) we see that the mutual coherence matrix is Hermitian in the sense that

$$
\mathcal{E}\left(\mathbf{r}_{1}, \mathbf{r}_{2}, \tau\right)=\mathcal{E}^{\dagger}\left(\mathbf{r}_{2}, \mathbf{r}_{1},-\tau\right)
$$

where the dagger stands for the Hermitian adjoint. It also satisfies various non-negative definiteness conditions (Sec. 6.5 in Ref. [1]).

The polarization properties of the field are described at a single point by the equal-time coherence matrix (or polarization matrix) obtained from Eq. (1) as

$$
\mathbf{J}(\mathbf{r})=\mathcal{E}(\mathbf{r}, \mathbf{r}, 0) .
$$

This matrix is (purely) Hermitian, i.e.,

$$
\mathbf{J}(\mathbf{r})=\mathbf{J}^{\dagger}(\mathbf{r}) .
$$

Next we introduce three quantities that are useful in characterizing electromagnetic coherence. The degree of polarization of beamlike fields is an old concept that describes the ratio of the intensity in the polarized part of the field to that of the total field [1]. Equally well it can be interpreted as a measure for the strength of correlation of the orthogonal electric field components at a single point in the frame where these components have equal intensities [2]. The degree of polarization $P(\mathbf{r})$ is defined via

$$
P^{2}(\mathbf{r})=1-\frac{4 \operatorname{det} \mathbf{J}(\mathbf{r})}{\operatorname{tr}^{2} \mathbf{J}(\mathbf{r})}=2 \frac{\operatorname{tr} \mathbf{J}^{2}(\mathbf{r})}{\operatorname{tr}^{2} \mathbf{J}(\mathbf{r})}-1,
$$

where det and tr denote the determinant and trace of the matrix, respectively.

The electromagnetic degree of coherence is a quantity that characterizes the strength of modulation of the (four) Stokes parameters in Young's interference experiment [5] or, equivalently, the strength of correlation of the field components at two points [4]. Although the analysis in Ref. [5] is done in the space-frequency domain, it is obvious that an analogous result holds in the space-time domain. The electromagnetic degree of coherence $\gamma_{\mathrm{EM}}\left(\mathbf{r}_{1}, \mathbf{r}_{2}, \tau\right)$, is written in the squared form as

$$
\gamma_{\mathrm{EM}}^{2}\left(\mathbf{r}_{1}, \mathbf{r}_{2}, \tau\right)=\frac{\operatorname{tr}\left[\mathcal{E}\left(\mathbf{r}_{1}, \mathbf{r}_{2}, \tau\right) \cdot \mathcal{E}\left(\mathbf{r}_{2}, \mathbf{r}_{1},-\tau\right)\right]}{\operatorname{tr}\left[\mathcal{E}\left(\mathbf{r}_{1}, \mathbf{r}_{1}, 0\right)\right] \operatorname{tr}\left[\mathcal{E}\left(\mathbf{r}_{2}, \mathbf{r}_{2}, 0\right)\right]}
$$

The fringe visibility in electromagnetic Young's interference experiment has also been referred to as the degree of coherence $[10,11]$. This quantity, denoted by $\gamma_{W}\left(\mathbf{r}_{1}, \mathbf{r}_{2}, \tau\right)$, is given by

$$
\gamma_{W}^{2}\left(\mathbf{r}_{1}, \mathbf{r}_{2}, \tau\right)=\frac{\operatorname{tr}^{2}\left[\mathcal{E}\left(\mathbf{r}_{1}, \mathbf{r}_{2}, \tau\right)\right]}{\operatorname{tr}\left[\mathcal{E}\left(\mathbf{r}_{1}, \mathbf{r}_{1}, 0\right)\right] \operatorname{tr}\left[\mathcal{E}\left(\mathbf{r}_{2}, \mathbf{r}_{2}, 0\right)\right]}
$$

From now on we consider the field at a single point $\left(\mathbf{r}_{1}=\mathbf{r}_{2}\right.$ $=\mathbf{r}$ ) and, for notational reasons, omit the explicit position dependence from the equations. For example, we write $\gamma_{\mathrm{EM}}^{2}(\mathbf{r}, \mathbf{r}, \tau)=\gamma_{\mathrm{EM}}^{2}(\tau)$, etc.

\section{DEFINITION OF POLARIZATION TIME}

Consider a monochromatic field, i.e., a field which contains only a single frequency component and hence is fully deterministic. For such a field the electric vector draws as a function of time a fixed ellipse, which may reduce to a line or a circle, and the field is fully polarized. In this case, we may say that the polarization state is static, although it may vary from point to point.

For polychromatic electromagnetic fields, deterministic or random, the instantaneous polarization ellipse can rotate in the plane orthogonal to the propagation direction and the magnitudes of the minor and major axes may change. Hence, the instantaneous polarization state and the corresponding polarization ellipse can be different at different instants of time, and we may say that the field exhibits polarization dynamics. We emphasize that the changes in the polarization ellipse can occur on a time scale shorter than the time required by the electric vector to make a complete cycle. When the polarization ellipse varies in time in a completely random manner, i.e., so that no preferred state of polarization exists when averaged over a long period of time, the field is fully unpolarized [3]. Note, however, that for any field at sufficiently short time interval no significant change in the polarization state takes place and, therefore, even a fully unpolarized field when considered within a short enough time interval is polarized. How short this time interval is, or after what duration the instantaneous polarization state of a fluctuating field has essentially changed? The aim of this work is to provide an answer to this question.

The mutual coherence matrix and the polarization matrix (1) and (3), respectively, cannot directly provide any information on the polarization dynamics as they consist of quantities which are time averages over a period that is much longer than the time scales characterizing the fluctuating optical field [3]. In order to access the polarization dynamics, we define the instantaneous Stokes parameters $S_{i}(t)$ with $i$ $=0, \ldots, 3$, as follows:

$$
\begin{gathered}
S_{0}(t)=E_{x}^{*}(t) E_{x}(t)+E_{y}^{*}(t) E_{y}(t), \\
S_{1}(t)=E_{x}^{*}(t) E_{x}(t)-E_{y}^{*}(t) E_{y}(t), \\
S_{2}(t)=E_{y}^{*}(t) E_{x}(t)+E_{x}^{*}(t) E_{y}(t), \\
S_{3}(t)=i\left[E_{y}^{*}(t) E_{x}(t)-E_{x}^{*}(t) E_{y}(t)\right] .
\end{gathered}
$$

These parameters are real quantities and when averaged over an infinite time interval, they reduce to the conventional Stokes parameters employed in the analysis of partial polarization [3]. It is straightforward to verify that the instantaneous Stokes parameters satisfy the relation

$$
S_{1}^{2}(t)+S_{2}^{2}(t)+S_{3}^{2}(t)=S_{0}^{2}(t),
$$

which reflects the fact that any field has instantaneously a certain polarization state, although it may vary as a function of time. In terms of the instantaneous Stokes parameters, we can introduce the instantaneous Poincaré vector [3] 


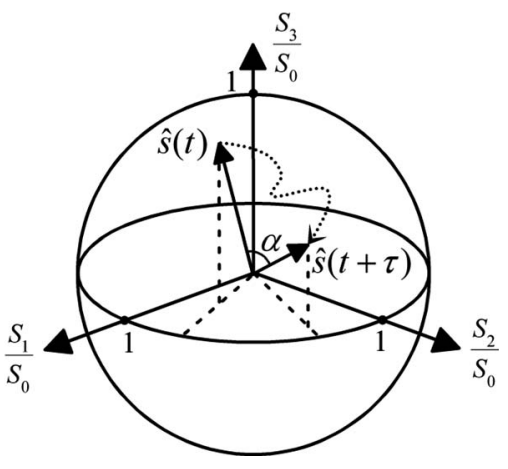

FIG. 1. Description of the instantaneous polarization state by a point on the Poincaré sphere of unit radius. The vectors $\hat{\mathbf{s}}(t)$ and $\hat{\mathbf{s}}(t+\tau)$ represent the polarization states at two instants of time with time difference $\tau$, and $\cos \alpha$ equals the scalar product $\hat{\mathbf{s}}(t) \cdot \hat{\mathbf{s}}(t+\tau)$.

$$
\mathbf{S}(t)=\left(S_{1}(t), S_{2}(t), S_{3}(t)\right),
$$

whose length is equal to the instantaneous intensity $S_{0}(t)$ as indicated by Eq. (12). It is insightful to express the above vector in the form

$$
\mathbf{S}(t)=\hat{\mathbf{s}}(t) S_{0}(t),
$$

where $\hat{\mathbf{s}}(t)$ is a unit vector parallel to $\mathbf{S}(t)$. Thus, we can interpret the Poincaré vector in Eq. (14) as a product of two quantities; a vector and a scalar that, respectively, represent the instantaneous polarization state (uniquely) and intensity. The vector $\hat{\mathbf{s}}(t)$ is conventionally used to express the state by a point on the Poincaré sphere of unit radius (see Fig. 1) [3]. If the polarization state fluctuates, the associated point on the surface of the Poincaré sphere traces a path as a function of time. If the instantaneous polarization state does not change, the point on the Poincaré sphere does not move. However, the intensity $S_{0}(t)$ of the field may fluctuate. Note also that if the point moves such that $\hat{\mathbf{s}}(t)$ and $\hat{\mathbf{s}}(t+\tau)$ are antiparallel, the related polarization states are orthogonal. For defining a time interval over which the instantaneous polarization state does not essentially change, we observe how far, on average, a point on the Poincaré sphere travels from its original position within a time interval $\tau$.

In order to characterize the fluctuation-induced change of the instantaneous polarization state, we introduce the following fourth-order (polarization) correlation function

$$
\begin{gathered}
C(\tau)=\langle\mathbf{S}(t) \cdot \mathbf{S}(t+\tau)\rangle \\
=\left\langle[\hat{\mathbf{s}}(t) \cdot \hat{\mathbf{s}}(t+\tau)] S_{0}(t) S_{0}(t+\tau)\right\rangle .
\end{gathered}
$$

The above function is a building block for constructing a measure for the statistical similarity of the instantaneous polarization states at times $t$ and $t+\tau$. From the lower row we see that the scalar product accounts for the difference in the instantaneous polarization states: it equals 1 if the states are the same and -1 if they are orthogonal. More precisely, the higher the value of the scalar product is, the closer to each other the associated points on the Poincaré sphere are after a time interval $\tau$ (see Fig. 1), and the more similar the polarization states are. Furthermore, the polarization states are weighted by the instantaneous intensities. Without the weighting, those instants of time with low or even zero intensity, and hence of less physical significance, would provide an equal contribution to the value of $C(\tau)$ as those with high intensity. Finally, averaging over time $t$ in Eq. (16) includes information on the similarity of the instantaneous polarization states over an infinite number of time intervals of length $\tau$.

It is desirable to normalize the parameter $C(\tau)$ in Eq. (16). We see that $C(\tau)$ acquires its maximum value when the polarization state is constant, i.e., $\hat{\mathbf{s}}(t)$ is independent of time. In this case, $C(\tau)=\left\langle S_{0}(t) S_{0}(t+\tau)\right\rangle$. Thus, the normalized polarization correlation function for the characterization of polarization dynamics takes on the form

$$
\gamma_{P}(\tau)=\frac{\langle\mathbf{S}(t) \cdot \mathbf{S}(t+\tau)\rangle}{\left\langle S_{0}(t) S_{0}(t+\tau)\right\rangle} .
$$

This parameter is a measure for the similarity of the polarization states at times $t$ and $t+\tau$. Using Eq. (16) $\gamma_{P}(\tau)$ can be interpreted as an intensity weighted average of the scalar product $\hat{\mathbf{s}}(t) \cdot \hat{\mathbf{s}}(t+\tau)$, i.e., of the closeness of the points on the Poincaré sphere (see Fig. 1). Note that the effective degree of coherence that describes the coherence properties of a field within a certain area or volume has the same intensityweighting form as $\gamma_{P}(\tau)[12,13]$. Making use of Eq. (16), we see at once from Eq. (17) that

$$
\gamma_{P}(0)=1
$$

and

$$
-1 \leqslant \gamma_{P}(\tau) \leqslant 1 .
$$

Equation (18) expresses the fact that, instantaneously, the field has a certain polarization state. The upper and lower limits in Eq. (19) correspond to the cases where after a time difference $\tau$ the polarization states are the same and orthogonal, respectively.

So far the analysis has been general and valid for any planar, stationary electromagnetic field. Assuming next that the field obeys Gaussian statistics, the fourth-order correlation functions in Eq. (17) can be expressed in terms of the second order ones [1]. This fact implies, after some algebra, that

$$
\begin{aligned}
\langle\mathbf{S}(t) \cdot \mathbf{S}(t+\tau)\rangle= & \operatorname{tr}^{2} \mathbf{J}-4 \operatorname{det} \mathbf{J}-\operatorname{tr}[\mathcal{E}(\tau) \cdot \mathcal{E}(-\tau)] \\
& +2|\operatorname{tr} \mathcal{E}(\tau)|^{2}=\operatorname{tr}^{2} \mathbf{J}\left[P^{2}-\gamma_{\mathrm{EM}}^{2}(\tau)+2\left|\gamma_{W}(\tau)\right|^{2}\right]
\end{aligned}
$$

and that

$$
\left\langle S_{0}(t) S_{0}(t+\tau)\right\rangle=\operatorname{tr}^{2} \mathbf{J}+\operatorname{tr}[\mathcal{E}(\tau) \cdot \mathcal{E}(-\tau)]=\operatorname{tr}^{2} \mathbf{J}\left[1+\gamma_{\mathrm{EM}}^{2}(\tau)\right] .
$$

In the above equations, the parameters $P, \gamma_{\mathrm{EM}}(\tau)$, and $\gamma_{W}(\tau)$ are those defined in Eqs. (5)-(7), respectively. Making use of Eqs. (20) and (21), we can write Eq. (17) in the form

$$
\gamma_{P}(\tau)=\frac{P^{2}-\gamma_{\mathrm{EM}}^{2}(\tau)+2\left|\gamma_{W}(\tau)\right|^{2}}{1+\gamma_{\mathrm{EM}}^{2}(\tau)}
$$

For $\tau=0$ we find that 


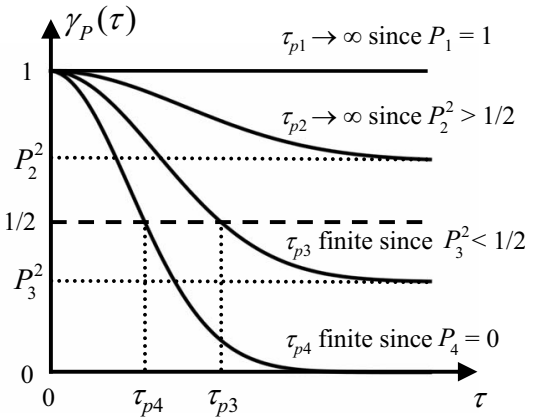

FIG. 2. Illustration of polarization time as discussed in the text. The topmost solid curve corresponds to a fully polarized field for which the polarization time is infinite. The second solid curve from the top represents a partially polarized field for which the polarization time is infinite as well. The third curve corresponds to a partially polarized field for which one can relate a polarization time $\tau_{p 3}$. The lowest curve corresponds to a fully unpolarized field for which the polarization time is $\tau_{p 4}$. The quantities $P_{i}$, with $i=1-4$, are the degrees of polarization related to the fields.

$$
\begin{gathered}
\gamma_{\mathrm{EM}}^{2}(0)=\frac{1}{2} P^{2}+\frac{1}{2}, \\
\gamma_{W}^{2}(0)=1,
\end{gathered}
$$

and, therefore, $\gamma_{P}(0)=1$ as was required by Eq. (18). Since the field is ergodic, the correlation between the electric fields vanishes as $\tau \rightarrow \infty$, and therefore, $\gamma_{\mathrm{EM}}(\tau) \rightarrow 0$ and $\gamma_{W}(\tau) \rightarrow 0$ in this limit, implying that

$$
\gamma_{P}(\tau) \rightarrow P^{2} \quad \text { when } \tau \rightarrow \infty \text {. }
$$

This result reflects the fact that when the field is partially polarized the instantaneous polarization states have, on average, a certain amount of similarity at any time difference $\tau$. This is intuitive since any beamlike field can be expressed as a sum of two uncorrelated fields; one is fully polarized and the other fully unpolarized. The polarized part is present at any time difference maintaining correlation of the field over two instants of time. Since all parameters in Eq. (22) involve only the elements of the equal-point mutual coherence matrix $\mathcal{E}(\tau)$ and the polarization matrix $\mathbf{J}, \gamma_{P}(\tau)$ can be measured by using interferometric techniques $[4,14]$, and polarization analysis $[1,15]$.

Next we define the polarization time, denoted henceforth by $\tau_{p}$, as a time difference over which the instantaneous polarization state does not significantly change. Mathematically, it can be defined by the criterion that $\gamma_{P}(\tau)$ decreases to some specific value, say $1 / 2$. This value is somewhat arbitrary and other appropriate values can be chosen as well. For our choice, the polarization time $\tau_{p}$ is defined by the condition $\gamma_{P}\left(\tau_{p}\right)=1 / 2$. Figure 2 illustrates qualitatively the definition of the polarization time. The topmost solid curve corresponds to a fully polarized field, for which the degree of polarization is $P_{1}=1$ and $\gamma_{P}(\tau)=1$ for all $\tau$. For this field, the polarization time is infinite since the state of polarization does not change (only the intensity can fluctuate). The two solid curves in the middle correspond to partially polarized fields. The degree of polarization of the upper one, $P_{2}$, satisfies $P_{2}^{2}>1 / 2$, and $\gamma_{P}(\tau)$ does not reach the value of $1 / 2$ for any $\tau$. Thus, in this case according to our criterium the polarization time is infinite as well. However, the degree of polarization $P_{3}$ of the lower one of the middle curves satisfies $P_{3}^{2}<1 / 2$ and therefore, at some time difference $\tau_{p 3}$, the function $\gamma_{P}(\tau)$ reaches the value of $1 / 2$. Thus, we may consider $\tau_{p 3}$ as being the time interval over which the instantaneous polarization state of this partially polarized field has not yet significantly changed. The lowest curve in Fig. 2 corresponds to a fully unpolarized field. For this field, the degree of polarization is zero $\left(P_{4}=0\right)$, and therefore, $\gamma_{P}(\tau)$ $\rightarrow 0$ when $\tau \rightarrow \infty$. The time difference $\tau_{p 4}$ at which $\gamma_{P}(\tau)$ $=1 / 2$ is the polarization time. The last case illustrates the fact that even for a fully unpolarized field, the instantaneous polarization state remains essentially unchanged within short enough a time interval.

The polarization time allows one to define the polarization length, i.e., a distance of propagation over which the polarization state remains effectively unaltered. In analogy with the relation between the coherence time and coherence length of scalar fields, we define the polarization length as

$$
l_{p}=c \tau_{p},
$$

where $c$ is the speed of light.

\section{EXAMPLES}

As the first example of the use of our formalism, we take a uniformly partially polarized, temporally Gaussian correlated beam propagating in the $z$ direction. The electric mutual coherence matrix of such a field can be written as

$$
\mathcal{E}(\tau)=\mathbf{J} \exp \left(-\tau^{2} / 2 \sigma^{2}\right),
$$

where $\mathbf{J}$ is the polarization matrix introduced in Eq. (3), and $\sigma$ characterizes the coherence time. The above coherence matrix satisfies the Hermiticity relation of Eq. (2), is nonnegative definite in the sense of Sec. 6.5 in Ref. [1], and the diagonal elements of $\mathbf{J}$ are non-negative since they are intensities. The matrix $\mathbf{J}$ contains all information about the polarization state at a single point, whereas the exponential term describes the temporal coherence properties. Using Eqs. (5)-(7) for the field characterized by Eq. (27), we obtain

$$
\begin{gathered}
\gamma_{\mathrm{EM}}^{2}(\tau)=\frac{1}{2}\left(1+P^{2}\right) \exp \left(-\tau^{2} / \sigma^{2}\right), \\
\gamma_{W}^{2}(\tau)=\exp \left(-\tau^{2} / \sigma^{2}\right) .
\end{gathered}
$$

Substituting the above two equations into Eq. (22) results in

$$
\gamma_{P}(\tau)=\frac{2 P^{2}+\left(3-P^{2}\right) \exp \left(-\tau^{2} / \sigma^{2}\right)}{2+\left(1+P^{2}\right) \exp \left(-\tau^{2} / \sigma^{2}\right)} .
$$

We see at once that in the limit of full polarization $(P \rightarrow 1)$ this formula reduces to $\gamma_{P}(\tau)=1$, and at $\tau \rightarrow \infty$ we obtain $\gamma_{P}(\tau) \rightarrow P^{2}$, as expected. Equation (30) is plotted in Fig. 3 for the degrees of polarization $P=0, P=0.5$, and $P=0.95$. The criterion that we use for the polarization time $\tau_{p}$ is $\gamma_{P}\left(\tau_{p}\right)$ $=1 / 2$. For $P=0.95$ the polarization time is infinite since $\gamma_{P}(\tau)>1 / 2$ for all $\tau$. In the cases of $P=0.5$ and $P=0$, the polarization times are $\tau_{p}=1.20 \sigma$ and $\tau_{p}=0.96 \sigma$, respectively 


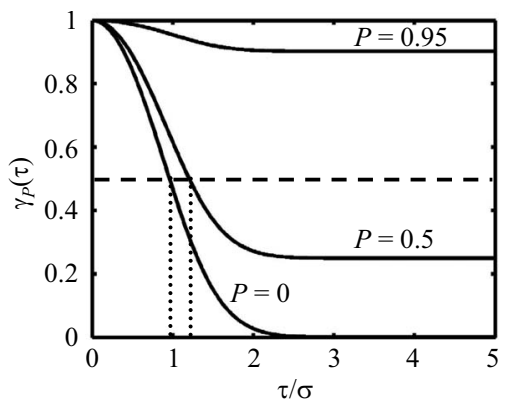

FIG. 3. Behavior of $\gamma_{P}(\tau)$ for a uniformly partially polarized, Gaussian correlated beam. The solid curves correspond to the degrees of polarization $P=0, P=0.5$, and $P=0.95$. The vertical dotted lines mark the polarization times, which are $\tau_{p}=0.96 \sigma$ and $\tau_{p}$ $=1.20 \sigma$ for $P=0$ and $P=0.5$, respectively.

(indicated with the dotted vertical lines). Thus, we conclude that when the field is weakly polarized and of the type represented by Eq. (27), the polarization time is close in value to the coherence time $\sigma$. Note, however, the difference between the behavior of the polarization time and the coherence time. When the degree of polarization increases the coherence time does not change, but the polarization time becomes longer and eventually infinite.

As a second example we consider the polarization time of the field radiated by a black-body source. By such a source we mean an aperture in a cavity containing a field in thermal equilibrium with the cavity walls. The aperture is assumed to be much larger than the mean wavelength of the field but so small that the equilibrium is not disturbed. The radiation properties of such a source are studied in Ref. [16], and it is found that the far field obeys Lambert's law and is fully unpolarized in all directions at all frequencies. Locally the radiated far field behaves as a plane wave, and at a distance $r$ in the direction specified by the unit vector $\hat{\mathbf{u}}$, the $2 \times 2$ (spectral) coherence matrix is given by [Eq. (21) in Ref. [16]]

$$
\boldsymbol{\Phi}(r \hat{\mathbf{u}}, \omega)=\frac{\mathcal{A} S_{0}(\omega) \cos \theta}{8 \pi r^{2}}\left(\begin{array}{ll}
1 & 0 \\
0 & 1
\end{array}\right) .
$$

In this equation, $\omega$ is the frequency, $\mathcal{A}$ represents the area of the aperture, and $\theta$ is the angle between the observation direction and the aperture normal. Furthermore, $S_{0}(\omega)$ is the Planck's spectrum, given by

$$
S_{0}(\omega)=\frac{\mathcal{B} \omega^{3}}{\exp \left(\hbar \omega / k_{B} T\right)-1},
$$

where $\mathcal{B}$ is a constant, $\hbar$ is the Planck's constant divided by $2 \pi, k_{B}$ is the Boltzmann constant, and $T$ is the temperature. According to the (generalized) Wiener-Khintchine theorem, the mutual coherence matrix and the cross-spectral density matrix constitute a Fourier transform pair (Sec. 2.4 in Ref. [1]). Making use of this fact, we find that the mutual coherence matrix is of the form

$$
\mathcal{E}(r \hat{u}, \tau)=\int_{0}^{\infty} \boldsymbol{\Phi}(r \hat{\mathbf{u}}, \omega) \exp (-i \omega \tau) d \omega,
$$

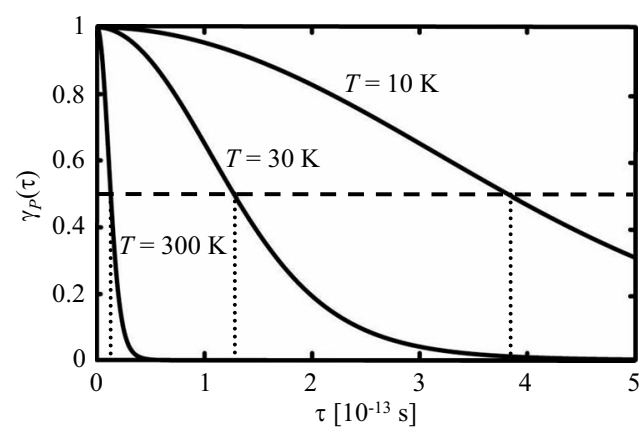

FIG. 4. Behavior of $\gamma_{P}(\tau)$ for black-body radiation at temperatures $T=10 \mathrm{~K}, T=30 \mathrm{~K}$, and $T=300 \mathrm{~K}$. The temperatures correspond to the polarization times $\tau_{p}=3.8 \times 10^{-13} \mathrm{~s}, \tau_{p}=1.3 \times 10^{-13} \mathrm{~s}$, and $\tau_{p}=1.3 \times 10^{-14} \mathrm{~s}$, respectively. The polarization times are marked with vertical dotted lines.

$$
=\frac{3 \mathcal{A B} \cos \theta}{4 \pi r^{2}}\left(\frac{k_{B} T}{\hbar}\right)^{4} \zeta\left(4,1+i \frac{k_{B} T}{\hbar} \tau\right)\left(\begin{array}{ll}
1 & 0 \\
0 & 1
\end{array}\right),
$$

where the lower limit of integration is zero due to the analytic signal representation of the electric field and $\zeta(s, a)$ is the generalized Riemann-Hurwitz zeta function [3].

Substituting Eq. (33) into Eqs. (5)-(7), one finds that

$$
\begin{gathered}
P=0, \\
\gamma_{\mathrm{EM}}(\tau)=\frac{90}{\sqrt{2} \pi^{4}}\left|\zeta\left(4,1+i \frac{k_{B} T}{\hbar} \tau\right)\right|, \\
\gamma_{W}(\tau)=\frac{90}{\pi^{4}} \zeta\left(4,1+i \frac{k_{B} T}{\hbar} \tau\right) .
\end{gathered}
$$

Note that the right-hand side of Eg. (36) is known as the Kano-Wolf formula obtained for the temporal coherence of black-body radiation [3,17]. Using the above functions in Eq. (22), we get for black-body radiation

$$
\gamma_{P}(\tau)=\frac{3\left|\frac{90}{\pi^{4}} \zeta\left(4,1+i \frac{k_{B} T}{\hbar} \tau\right)\right|^{2}}{2+\left|\frac{90}{\pi^{4}} \zeta\left(4,1+i \frac{k_{B} T}{\hbar} \tau\right)\right|^{2}},
$$

which depends only on the temperature, as expected. The behavior of $\gamma_{P}(\tau)$ at temperatures $T=10 \mathrm{~K}, T=30 \mathrm{~K}$, and $T$ $=300 \mathrm{~K}$ is depicted in Fig. 4. The polarization times at these temperatures are obtained with the criterion $\gamma_{P}\left(\tau_{p}\right)=1 / 2$ and they, respectively, have the values of $\tau_{p}=3.8 \times 10^{-13} \mathrm{~s}, \tau_{p}$ $=1.3 \times 10^{-13} \mathrm{~s}$, and $\tau_{p}=1.3 \times 10^{-14} \mathrm{~s}$ (marked with vertical dotted lines). We see that when the temperature decreases the polarization time becomes longer. In fact, in the limit $T \rightarrow 0$ we find that $\gamma_{P}(\tau) \rightarrow 1$, indicating that the polarization time asymptotically approaches infinity when the temperature decreases. Making use of Eq. (26) and the above values for the polarization times at the three temperatures, one can determine the corresponding polarization lengths, $l_{p}$, which are $l_{p}=114 \mu \mathrm{m}, l_{p}=39 \mu \mathrm{m}$, and $l_{p}=3.9 \mu \mathrm{m}$, respectively.

The explicit dependence of the polarization length on temperature is obtained by solving for the product $T \tau_{p}$ from 
Eq. (37) with the condition $\gamma_{p}\left(\tau_{p}\right)=1 / 2$, and using Eq. (26). The result is

$$
l_{p}=\frac{a}{T},
$$

where $a=1.14 \times 10^{-3} \mathrm{Km}$. The cosmic microwave background $(\mathrm{CMB})$ radiation is known to be a very accurate black-body field at the temperature $T=2.73 \mathrm{~K}[18]$. For this field one obtains $l_{p, \mathrm{CMB}}=0.42 \mathrm{~mm}$. Another example of black-body field is the thermal radiation from the Sun, whose surface temperature is $T=5800 \mathrm{~K}$. The corresponding polarization length has the value of $l_{p, \text { Sun }}=200 \mathrm{~nm}$.

\section{SUMMARY}

We investigate the polarization dynamics of stationary, random electromagnetic beams, and introduce an intensitynormalized polarization correlation function that describes the correlation of the Poincaré vectors, i.e., the statistical similarity of instantaneous polarization states, at a certain time difference. The correlation function is used to define a polarization time and related polarization length over which the instantaneous polarization state does not essentially change. In the case of Gaussian statistics, the polarization correlation function is found to assume a simple form in terms of measurable quantities that describe the partial electromagnetic coherence and partial polarization of an optical beam. The formalism is applied to a uniformly partially polarized, temporarily Gaussian correlated beam and radiation from an opening of a black-body cavity. The results are expected to be useful in cases when the polarization fluctuations play a significant role, e.g., in studies on light-matter interaction [19], semiconductor lasers [20], supercontinuum generation [21], and cosmology [22].

\section{ACKNOWLEDGMENTS}

This work was supported by the Academy of Finland (118331). A. T. Friberg also acknowledges funding from the Swedish Foundation for Strategic Research (SSF).
[1] L. Mandel and E. Wolf, Optical Coherence and Quantum Optics (Cambridge University Press, Cambridge, UK, 1995).

[2] M. Born and E. Wolf, Principles of Optics, 7th ed. (Cambridge University Press, Cambridge, England, 1999).

[3] C. Brosseau, Fundamentals of Polarized Light: A Statistical Optics Approach (Wiley, New York, 1998).

[4] J. Tervo, T. Setälä, and A. T. Friberg, Opt. Express 11, 1137 (2003).

[5] T. Setälä, J. Tervo, and A. T. Friberg, Opt. Lett. 31, 2669 (2006).

[6] E. Wolf, Introduction to the Theory of Coherence and Polarization of Light (Cambridge University Press, Cambridge, UK, 2007).

[7] T. Setälä, A. Shevchenko, M. Kaivola, and A. T. Friberg, Phys. Rev. E 66, 016615 (2002).

[8] C. Brosseau and A. Dogariu, Prog. Opt. 40, 315 (2006).

[9] J. J. Gil, J. Appl. Phys. 40, 1 (2007).

[10] B. Karczewski, Phys. Lett. 5, 191 (1963).
[11] E. Wolf, Phys. Lett. A 312, 263 (2003).

[12] P. Vahimaa and J. Tervo, J. Opt. A, Pure Appl. Opt. 6, S41 (2004).

[13] K. Blomstedt, T. Setälä, and A. T. Friberg, J. Opt. A, Pure Appl. Opt. 9, 907 (2007).

[14] F. Gori, Opt. Lett. 23, 241 (1998).

[15] K. Lindfors et al., Nat. Photonics 1, 228 (2007).

[16] D. F. V. James, Opt. Commun. 109, 209 (1994).

[17] Y. Kano and E. Wolf, Proc. Phys. Soc. London 80, 1273 (1962).

[18] R. H. Dicke, P. J. E. Peebles, P. G. Roll, and D. T. Wilkinson, Astrophys. J. 142, 414 (1965).

[19] A. Shevchenko, M. Kaivola, and J. Javanainen, Phys. Rev. A 73, 035801 (2006).

[20] H. F. Hofmann and O. Hess, Quantum Semiclassic. Opt. 10, 87 (1998)

[21] Z. Zhu and T. G. Brown, Opt. Express 12, 791 (2004).

[22] P. Coles, Nature (London) 433, 248 (2005). 